\title{
Хроматографическое поведение монокофеоилхинных и дикофеоилхинных кислот в условиях ОФ ВЭЖХ: зависимость от строения
}

\author{
(C) 2021 Дейнека В.И., Олейниц Е.Ю., Дейнека Л.А. \\ Белгородский государственный национальный исследовательский университет, Белгород
}

Поступила в редакцию 5.05.2021 г.

DOI: $10.17308 /$ sorpchrom.2021.21/3628

Определена зависимость удерживания изомерных хлорогеновых кислот (монокофеоилхинных и дикофеоилхинных) от положения ацилирования хинной кислоты в условиях обращеннофазовой ВЭЖХ. В работе использованы экстракты листьев Ilex paraguariensis (чай мате) и соцветий Cynara scolymus (бутоны артишока) с известным составом хлорогеновых кислот. Показано, что на картах разделения дикофеоилхинные кислоты (diCQAs) имеют линии тренда с характеристическим параметром $a$ относительного удерживания по уравнению $\lg k(\mathrm{i})=a \cdot \lg k($ кофейная кислота $)+b$. Параметр $a$ для diCQAs примерно вдвое больше, чем для монокофеолихинных кислот (CQAs). По величине параметра $a$ возможно обнаружение изомеров diCQAs с дополнительным использованием УФспектра, характерного только среди остальных экстрактивных веществ фенольной природы, без массспектрометрического детектирования. Исключением является 1,3-дикофеоилхинная кислота (1,3diCQA), у которой наблюдается внутримолекулярная копигментация за счет стэкинг взаимодействий ароматических колец, находящихся в близко расположенных аксиальных положениях в одной из конформаций молекулы. Такая структура найдена при оптимизации геометрии методом MM2 в программе Chem3D. Вследствие возникновения стэкинга сорбция 1,3DiCQA одновременно двумя радикалами кофейной кислоты затруднена, поэтому параметр а принимает существенно меньшее значение (1.674). Внутримолекулярный стэкинг подтвержден изменением электронного спектра поглощения 1,3diCQA по сравнению со спектрами остальных хлорогеновых кислот.

В работе показано, что в случае дикофеоилхинных кислот возможны инверсии удерживания в ряду 3.4diCQA-3,5diCQA-1.5diCQA кислот при изменении состава подвижной фазы. При этом дифференциация между этими изомерами и 4,5diCQA возможна сопоставлением параметра $a$ уравнения линии тренда относительного удерживания этих кислот и монокофеоилхинных кислот в предположении отсутствия существенных внутримолекулярных структурных эффектов, влияющих на удерживание diCQA. При исследовании зависимости удерживания diCQAs от pH подвижных фаз установлено, что возможна дифференциация изомеров положения кислот по двум параметрам: по кислотности карбоксильной группы хинной кислоты (более высокой кислотностью обладают изомеры, включающие ацилирование по положению 1), по степени падения времени удерживания, которая оказывается наивысшей для изомеров, содержащих ацильную группу в положении 5.

Ключевые слова: монокофеолихинные кислоты, дикофеоилхинные кислоты, параметры уравнения относительного удерживания, удерживание в условиях ОФ ВЭЖХ, зависимость от состава элюента, зависимость от рН элюента, зависимость от строения.

\section{Введение}

Характерная черта большинства современных исследований состава экстрактов методом ВЭЖХ - недооценка значимости хроматографического поведе- ния веществ при их определении, которая во многих случаях заменяется использованием большого набора стандартных соединений. Так, например, при разработке способа дифференциации кофеоилхинных кислот тщательно изучены возможности использования 
направления фрагментации при массспектрометрическом детектировании, а информация об удерживании изомеров рассматривалась лишь как вспомогательный фактор [1]. Причем основное внимание в цитированной работе уделялось порядку элюирования, что нельзя признать корректным подходом, поскольку порядок элюирования может зависеть от состава подвижной фазы (или профиля градиента), используемого при разделении веществ.

Между тем хроматографическое поведение веществ является строгой функцией не только свойств стационарной и подвижной фаз, но и строения соединений. Так, например, при применении метода анализа относительного удерживания антоцианов было установлено, что наклоны линий тренда последовательно возрастают примерно на 0.1 при усложнении гликозидного заместителя добавлением второго или третьего моносахарида $[2,3]$, что позволяет с использованием только хроматографии отличать 3 моногликозиды от 3-ди- и 3тригликозидов без использования массспектрометрического детектирования.

Дикофеоилхинные кислоты часто синтезируются в различных видах растений наряду с монокофеоилхинными кисло- тами [4]. Как производные кофейной кислоты, содержащие две ортогидроксильные группы в каждом из радикалов, они обладают высокой антиоксидантной активностью. В структуре хинной кислоты имеются четыре гидроксильные группы, поэтому возможно существование четырех изомеров. Согласно ИЮПАК [5], необходимо проводить нумерацию атом углерода в цикле, как указано на рис. 1.

При такой нумерации неохлорогеновая кислота - это 3-кофеоилхинная кислота (3СQА), хлорогеновая - 5-кофеоилхинная (5CQA), а криптохлорогеновая 4-кофеоилхинная (4CQA). В экстрактах некоторых растений обнаружена и 1кофеоилхинная кислота (1CQA). При этом возможно существование шести изомерных дикофеоилхинных кислот: 1,3diCQA, 1,4diCQA, 1,5diCQA, 3,4diCQA, 3,5diCQA и 4,5diCQA. Из зеленого кофе были выделены три последних изомера [6], они же синтезируются в листьях Ilex paraguariensis (Maté) [7], a первый и третий изомеры обнаружены (с другими дикофеоилхинными кислотами), например, в артишоке, Cynara scolymus [8].

Цель настоящей работы - установление закономерностей хроматографического

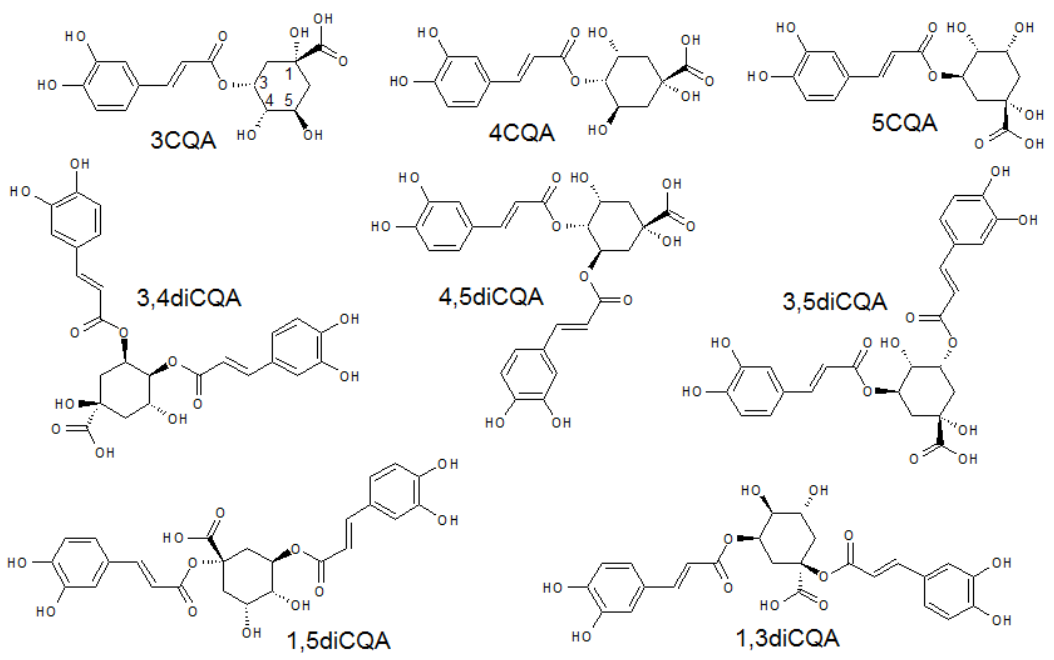

Рис. 1. Нумерация атомов углерода циклогексанового цикла хинной кислоты и изомеры хлорогеновых кислот

Fig. 1. Numbering of carbon atoms in the cyclohexane cycle of quinic acid and isomers of chlorogenic acids 


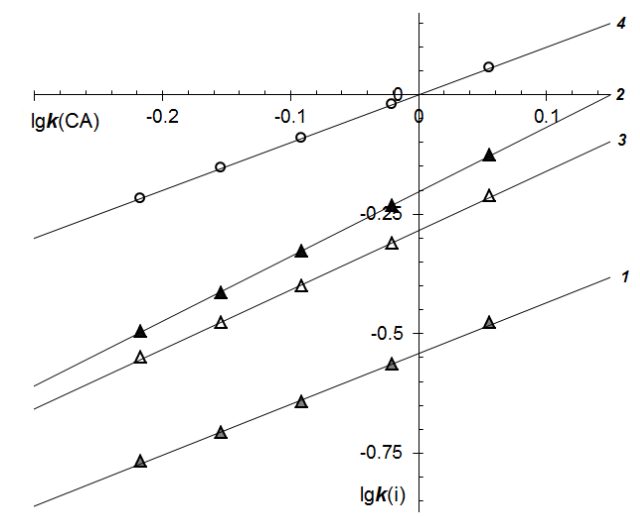

Рис. 2. Карта разделения монокофеоилхинных кислот. Вещества: 1 - 3CQA; 2 - 4CQA; 3 - 5CQA; 4 - СА (кофейная кислота)

Fig. 2. A separation map of mono-caffeoylquinic acids. Substances: 1 -3CQA; 2 -4CQA; 3 - 5CQA; 4 - CA (caffeic acid)

поведения дикофеоилхинных кислот для идентификации этих изомеров без применения труднодоступных экспериментальных методов.

\section{Экспериментальная часть}

Экстракты были получены из чая мате («Чайная коллекция», ООО «РЧКТрейдинг»), из консервированных соплодий артишока и из препарата «Артишока экстракт» (ЗАО «Эвалар») настаиванием в кипящей воде в течение 30 мин. Для хроматографических исследований использовали экстракты, очищенные методом твердофазной экстракции на концентрирующих патронах ДИАПАК С18. В работе в качестве стандартных соединений использовали 5кофеоилхинную (SIGMA-ALDRICH, India) и кофейную (Alfa Aesar, USA) кислоты.

В работе хроматографическое поведение хлорогеновых кислот исследовали, используя хроматограф Agilent 1200 Infinity с диодно-матричнымо детектором. Разделение выполняли на колонке $150 \times 4.6$ мм Kromasil 300-5-С4 при температуре термостата колонок $30^{\circ} \mathrm{C}$. Все эксперименты выполняли в изократическом варианте элюирования с подвижными фазами вода-0.25 об.\% $\mathrm{H}_{3} \mathrm{PO}_{4}-\phi$ об.\% $\mathrm{CH}_{3} \mathrm{CN}$, где $\phi$ - содержание ацетонитрила, которое выбирали из диапазона 12.4-17.2 об.\%. Мертвое время опреде- ляли по удерживанию щавелевой кислоты.

Подвижные фазы готовили из дистиллированной воды, 0.25 об.\% ортофосфорной кислоты (РЕАХИМ, Россия) и ацетонитрила (Fisher Chemical, UK). После смешивания компонентов полученный раствор профильтровывали и дегазировали.

Минимизацию энергии и определение геометрии молекул выполняли в программе Chem3D (PerkinElmer) методом MM2.

\section{Обсуждение результатов}

Карта разделения монокофеоилхинных кислот в использованной хроматографической системе по методу анализа относительного удерживания представлена на рис. 2. В этой карте в качестве реперного соединения использована кофейная кислота:

$$
\lg k(\mathrm{i})=a \cdot \operatorname{lgk}(\mathrm{CA})+b,
$$

где $\mathrm{k}$ - факторы удерживания веществ, СА - кофейная (3,4-дигидроксикоричная) кислота.

Параметры уравнений линий трендов на рис. 2 представлены в табл. 1 .

Параметр $a$ в уравнении (1) указывает на соотношение количества молекул $\mathrm{CH}_{3} \mathrm{CN}$, высвобождаемых при сорбции соответствующей хлорогеновой кислоты по отношению к кофейной кислоте. По карте разделения видно, что в выбранной хроматографической системе 
Таблица 1. Параметры уравнения (1) для монокофеоилхинных кислот

Table 1. Parameters of equation (1) for mono-caffeoylquinic acids

\begin{tabular}{|c|c|c|c|c|}
\hline \multirow{2}{*}{$№$} & \multirow{2}{*}{ Монокофеоилхинная кислота } & \multicolumn{2}{|c|}{ Параметры уравнения (1) } & \multirow{2}{*}{$\mathrm{R}^{2}$} \\
\cline { 3 - 4 } & & $a$ & $b$ & \\
\hline 1 & 3-кофеоилхинная кислота, 3СQА & 1.064 & -0.540 & 0.9986 \\
\hline 2 & 4-кофеоилхинная кислота, 4СQА & 1.353 & -0.203 & 0.9998 \\
\hline 3 & 5-кофеоилхинная кислота, 5СQА & 1.243 & -0.283 & 0.9994 \\
\hline
\end{tabular}

(в отличие от некоторых других систем, [9]) порядок элюирования хлорогеновых кислот постоянен в любом приемлемом (при котором фактор удерживания первого компонента не меньше 1 , а последнего - не больше 20) составе подвижной фазы:

$$
\mathrm{t}_{\mathrm{R}}(3 \mathrm{CQA})<\mathrm{t}_{\mathrm{R}}(5 \mathrm{CQA})<\mathrm{t}_{\mathrm{R}}(4 \mathrm{CQA}),
$$

и только при использовании элюентов с относительно большой элюирующей силой возможна инверсия времен удерживания двух последних соединений, но при этом их удерживание будет близко к мертвому времени. Время удерживания кофейной кислоты медленнее возрастает с уменьшением элюирующей силы подвижной фазы по сравнению с тремя изомерными хлорогеновыми кислотами, поэтому включение этого соединения в представленный выше порядок элюирования невозможно, вследствие зависимости порядка элюирования от состава подвижной фазы. В таком случае удобно воспользоваться временем удерживания

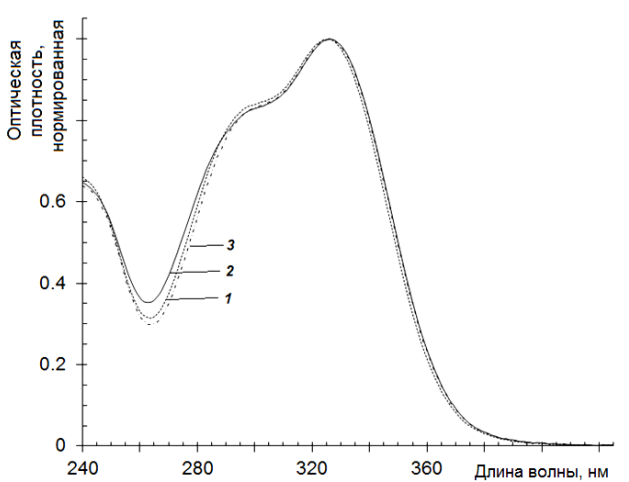

Рис. 3. Нормированные электронные спектры поглощения монокофеоилхинных кислот

1 - 3CQA; 2 - 4CQA; 3 - 5CQA

Fig. 3. Normalised electronic absorption spectra of mono-caffeoylquinic acids 1 - 3CQA; 2 - 4CQA; 3 - 5CQA веществ для подвижных фаз, найденных по экстраполяционному уравнению [10]:

$$
\lg k(\mathrm{i})=a_{0}-a_{1} \cdot \phi+a_{2} \cdot \phi^{2}
$$

где $\phi$ - объемная доля $\mathrm{CH}_{3} \mathrm{CN}$ в подвижной фазе. В таком случае порядок становится однозначным для выбранной хроматографической системы:

$$
a_{0}(3 \mathrm{CQA})=0.991<a_{0}(\mathrm{CA})=1.120<
$$

$a_{0}(5 \mathrm{CQA})=1.360<a_{0}(4 \mathrm{CQA})=1.456$.

Очевидно, что наибольший наклон линий тренда (по уравнению 1) характерен для 4CQA, заметно меньшим оказывается этот параметр для 5CQA, а для 3CQA $a$ принимает наименьшее значение, оставаясь больше 1.000 , табл. 1.

При идентификации монокофеоилхинных кислот использовали подход, предложенный в работе [9], с контролем по параметрам электронных спектров поглощения, близких для трех изомерных кислот, но заметно отличающихся от спектра самой кофейной кислоты, рис. 3.

Карта разделения дикофеоилхинных кислот с тем же реперным соединением,

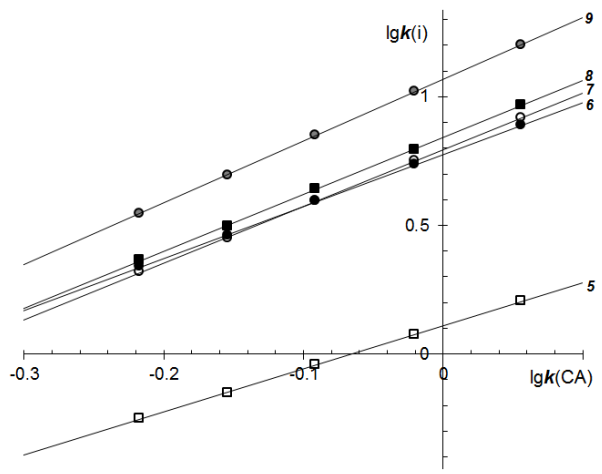

Рис. 4. Карта разделения дикофеоилхинных кислот. Вещества: 4 - 1,3diCQA; 5 -3,5diCQA; 6 -3,4diCQA; 7 - 1,5diCQA; $8-4,5$ diCQA

Fig. 4. A separation map of dicaffeoylquinic acids. Substances: 4 - 1,3diCQA; 5 - 3,5diCQA; 6 -3,4diCQA; $7-1.5 \mathrm{diCOA}: 8-4.5 \mathrm{diCOA}$ 
Таблица 2. Параметры уравнения (1) для дикофеоилхинных кислот

Table 2. Parameters of equation (1) for di-caffeoylquinic acids

\begin{tabular}{|l|c|c|c|c|}
\hline \multirow{2}{*}{ № } & \multirow{2}{*}{ Монокофеоилхинная кислота } & \multicolumn{2}{|c|}{ Параметры уравнения (1) } & \multirow{2}{*}{$\mathrm{R}^{2}$} \\
\cline { 3 - 4 } & & $a$ & $b$ & \multirow{2}{*}{0.99974} \\
\hline 6 & 1,3-дикофеоилхинная кислота, 1,3diCQA & 1.674 & 0.111 & 0.999984 \\
\hline 7 & 3,5-дикофеоилхинная кислота, 3,5diCQA & 2.019 & 0.778 & 0.999 \\
\hline 8 & 3,4-дикофеоилхинная кислота, 3,4diCQA & 2.203 & 0.795 & 0.99988 \\
\hline 9 & 4,5-дикофеоилхинная кислота, 3,5diCQA & 2.217 & 0.843 & 0.99981 \\
\hline
\end{tabular}

представлена на рис. 4.

Как видно из данных табл. 2 параметр $a$ линий трендов четырех из пяти обнаруживаемых на хроматограммах дикофеоилхинных кислот почти в 2 раза больше, чем в случае монокофеоилхинных кислот. Только для 1,3-дикофеоилхинной кислоты как параметр $a$, так и удерживание оказываются наименьшими. Причина указанного свойства связана с особенностями строения этого изомера. И действительно, минимизация энергии (в вакууме) геометрии 1,3diCQA методом молекулярной механики MM2 (в вакууме) в программе Chem3D привела к структуре, в которой наблюдается взаимодействие ароматических систем обоих заместителей, находящихся в аксиальных (по отношению к С6-кольцу) положениях, рис. 5. Это подтверждается заметным изменением УФ спектра изомера по сравнению со всеми остальными изомерами, рис. 6.

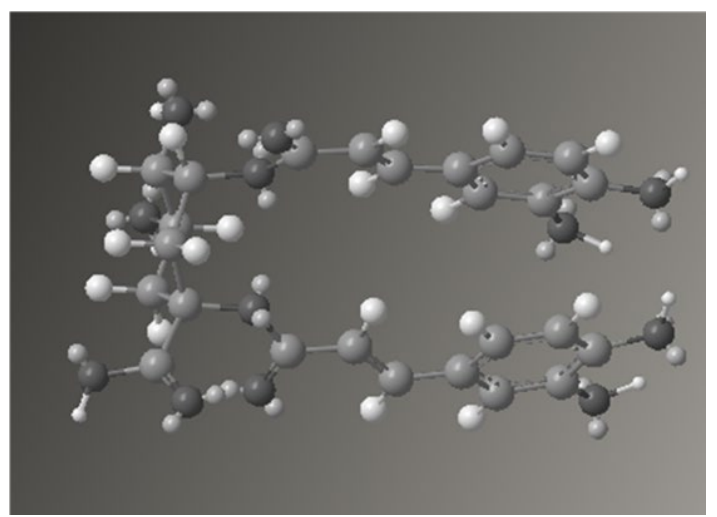

Рис. 5. Одна из возможных конформаций 3,5diCQA

Fig. 5. A possible 3,5diCQA conformation
Для остальных изомеров можно предположить одновременную сорбцию соединений обоими радикалами кофейной кислоты, хотя итоговые параметры $a$ для всех изомеров несколько меньше суммы параметров $a$ для соответствующих монокофеоилхинных кислот. Впрочем, дифференциация дикофеоилхинных от монокофеоилхинных кислот не требует применения масс-спектрометрического детектирования при близких электронных спектрах поглощения.

Дифференциация между изомерными кислотами может быть выполнена по анализу параметров $a$ : самый большой наклон линий трендов должен соответствовать 4,5diCQA, как веществу с наибольшей суммой параметров $a$ для индивидуальных 4CQA и 5CQA. Далее наклон линий трендов должен уменьшиться для 3,4diCQA, за которой последует 3,5diCQA. 1,5-diCQA может быть предположительно определена, как кис-

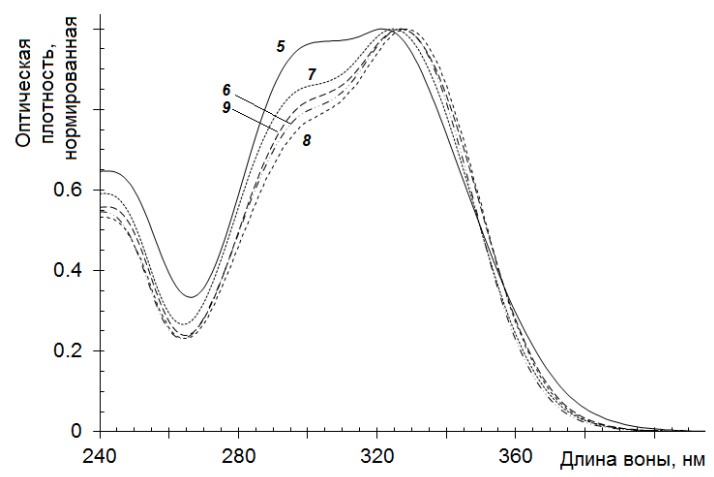

Рис. 6. Нормированные электронные спектры поглощения дикофеоилхинных кислот.

Вещества: 5 - 1,3diCQA; 6 - 3,5diCQA;

7 -3,4diCQA; 8 - 1,5diCQA; 9 - 4,5diCQA

Fig. 6. Normalised electronic absorption spectra of di-caffeoylquinic acids. Substances:

5 - 1,3diCQA; $6-3,5$ diCQA; $7-3,4$ diCQA; $8-1,5$ diCQA; $9-4,5$ diCQA 


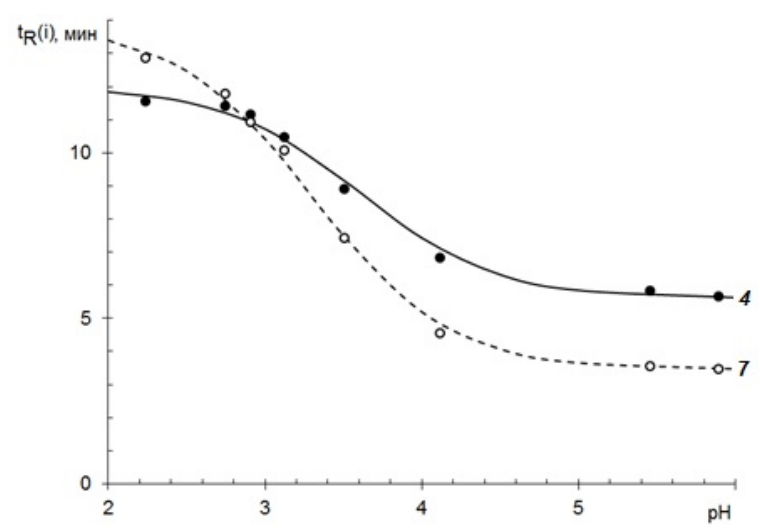

Рис. 7. Зависимость удерживания хлорогеновых кислот от рН. Вещества: 4 - 1,3diCQA; $7-1,5$ diCQA

Fig. 7. Dependence of the retention of chlorogenic acids on $\mathrm{pH}$. Substances: 4 - 1,3diCQA; 7 - 1,5diCQA

лота из экстракта артишока, которая отсутствовала в экстракте мате при двукратном росте параметра $a$ и близком к первым трем изомерам электронном спектре поглощения. Отметим, что если 4,5diCQA, имеющая наибольшее удерживание, легко отделяется от остальных изомеров, то три других изомера (3,5diCQA, 3,4diCQA и 1,5diCQA) имеют близкие времена удерживания, причем возможны инверсии порядка элюирования 3,5diCQA с двумя другими изомерами (3,4diCQA и 1,5diCQA), что объясняет различные последовательности элюирования этих соединений в известных публикациях [11-15]. Поэтому в качестве важной и однозначной характеристики порядка удерживания дикофеоилхинных кислот следует воспользоваться их удерживанием в подвижных фазах, не содержащих органического модификатора, определяемых по уравнению (2):

$$
\begin{gathered}
a_{0}(1,3 \mathrm{diCQA})=2.154<a_{0}(3,5 \mathrm{diCQA})= \\
3.109<a_{0}(3,4 \mathrm{diCQA})=3.345< \\
<a_{0}(1,5 \mathrm{diCQA})=3.479<a_{0}(4,5 \mathrm{diCQA}) \\
=3.696 .
\end{gathered}
$$

Подтверждение правильности отнесения 1,5diCQA было получено в другой серии экспериментов - по изучению зависимости удерживания хлорогеновых кислот при изменении $\mathrm{pH}$. В этих экспериментах было установлено, что падение удерживания хлорогеновых кислот с ростом $\mathrm{pH}$ выше 2 (условие практически полного подавления диссоциации карбоксильной группы) происходит несколько раньше в случае 1,3diCQA и 1,5diCQA, рис. 7. Это объясняется повышением кислотности карбоксильной группы при ацилировании ОН-группы в положении 1 , что позволяет дифференцировать такие изомеры от других 3,4diCQA, 3,5diCQA и 4,5diCQA. Кроме того, уменьшение факторов удерживания при росте $\mathrm{pH}$ для изомеров diCQA (как и CQAs) оказывается наибольшим при наличии радикала кофейной кислоты в положении 5, что было установлено на примере монокофеоилхинных кислот.

\section{Заключение}

Таким образом, параметр $a$ уравнения относительного удерживания с контролем по электронным спектрам поглощения позволяет идентифицировать дикофеоилхинные кислоты в сумме экстрактивных веществ без применения массспектрометрического детектирования. Среди изомерных дикофеоилхинных кислот дифференциация изомеров может быть выполнена по изменению удерживания при росте $\mathrm{pH}$ вследствие двух факторов: более высокой кислотности карбоксильной группы при ацилировании ОН-группы в положении 1 и большего падения удерживания изомеров, содержащих ОН-группу в положении 5. 
Работа поддержана грантом Российского фонда фундаментальных исследований «Аспиранты», номер 20-33-90031/20.

\section{Список литературы}

1. Clifford M.N., Knight S., Kuhnert N. // J. Agric. Food Chem. 2005. Vol. 53. pp. 38213832.

2. Дейнека В.И., Григорьев А.М. // Ж. аналит. химии. 2004. Т. 59. № 3. С. 305-309.

3. Deineka V.I., Deineka L.A., Saenko I.I. // J. Anal. Methods Chem. 2015. Vol .2015. ID 732918.

4. Clifford M.N. // J. Sci. Food Agric. 2000. Vol. 80. pp. 1033-1042.

5. IUPAC Commission on the Nomenclature of Organic Chemistry (CNOC) and IUPACIUB Commission on Biochemical Nomenclature (CBN). Nomenclature of cyclitols. Recommendations, 1973 // Biochem. J. 1976. Vol. 153. pp. 23-31.

6. Clifford M.N., Johnston K.L., Knight S. et al. // J. Agric. Food Chem. 2003. Vol. 51. pp. 2900-2911.

7. de Paula Lima J., Farah A., King B. et al. // J. Agric. Food Chem. 2016. Vol. 64. pp. 23612370.
8. Schütz K., Kammerer D., Carle R. et al. // J. Agric. Food Chem. 2004. Vol. 52. pp. 40904096

9. Дейнека В.И., Олейниц Е.Ю., Блинова И.П. и др. // Ж. аналит. химии. 2019. Т 74. № 8. C. $588-594$.

10. Schoenmakers P.J., Billiet H.A.H., Tijssen R. et al. // J. Chromatogr. A. 1978. Vol. 149. pp. 519-37.

11. Murugesu K., Saghir S.A.M., Sadikun A. et al. // Acta Chromatographica. 2021. Vol. 33. pp. 170-178.

12. Bicchi C.P., Binello A.E., Pellegrho G.M. et al. // J. Agric. Food Chem. 1995. Vol. 43. pp. 1549-1555.

13 Ky C.-L., Noirot M., Hamon S. // J. Agric. Food Chem. 1997. Vol. 45. pp. 786-790.

14. Xue M., Shi H., Zhang J. et al. // Molecules. 2016. Vol. 21. 948.

15. Stalmach A., Mullen W., Nagai C. et al. // Braz. J. Plant Physiol. 2006. Vol. 18. pp. 253262.

\title{
Chromatographic behaviour of mono-caffeoylquinic and di-caffeoylquinic acids under the conditions of reversed-phase HPLC: structural dependences
}

\author{
(C) 2021 Deineka V.I., Oleinits E.Yu., Deineka L.A. \\ Belgorod State National Research University, Belgorod
}

\begin{abstract}
The research determined the dependence of the retention of isomeric chlorogenic acids (monocaffeoylquinic and di-caffeoylquinic) on the position of quinic acid acylation under the conditions of reversed-phase HPLC. We used leaf extracts of Ilex paraguariensis (mate tea) and inflorescences of Cynara scolymus (artichoke buds) with a known composition of chlorogenic acids. It was shown that on separation maps di-caffeoylquinic acids (diCQAs) had trend lines with the characteristic parameter $a$ of the relative retention calculated using the equation $\lg k(\mathrm{i})=a \lg k$ (caffeic acid $)+b$. The parameter $a$ for diCQAs was about twice as much as for mono-caffeoylquinic acids (CQAs). Using the value of the parameter $a$ it is possible to detect diCQAs isomers with the additional use of the UV spectrum (which is only characteristic of other extractive substances of a phenolic nature) without mass spectrometric detection. An exception is 1,3-dicaffeoylquinic (1,3diCQA), which demonstrates intramolecular copigmentation due to the stacking interactions of aromatic rings located in closely spaced axial positions in one of the molecular conformations. Such a structure was found as a result of the geometry optimisation by the MM2 method and the Chem3D software. Due to stacking, the simultaneous sorption of 1,3DiCQA by two radicals of caffeic acid was impeded. Therefore, the parameter $a$ had a significantly smaller value (1.674). Intramolecular stacking was confirmed by a change in the electronic absorption spectrum of 1,3diCQA in comparison with the spectra of other chlorogenic acids.

It was shown that in case of di-caffeoylquinic acids, there is a possibility of retention inversions in the series of 3.4diCQA-3.5diCQA-1.5diCQA acids with a change in the composition of the mobile phase. In
\end{abstract}


this case, it is possible to differentiate between these isomers and 4,5diCQA by comparing the parameter $a$ in the equation for the trend line of the relative retention of these acids and mono-caffeoylquinic acids, assuming there are no significant intramolecular structural effects affecting the diCQA retention. The study of the dependence of the diCQAs retention on the $\mathrm{pH}$ of mobile phases revealed that it is possible to differentiate acid position isomers by two parameters: by the acidity of the carboxyl group of quinic acid (isomers with acylation at position 1 have higher acidity) and by the degree of decrease in the retention time, which is the highest for isomers with an acyl group at position 5 .

Keywords: mono-caffeoylquinic acids, di-caffeoylquinic acids, parameters of the equation for relative retention, retention under the conditions of the reversed-phase HPLC, dependence on the eluent composition, dependence on the $\mathrm{pH}$ of the eluent, dependence on the structure.

\section{References}

1. Clifford M.N., Knight S., Kuhnert N. J. Agric. Food Chem., 2005, Vol. 53, pp. 38213832. https://doi.org/10.1021/jf050046h

2. Deineka, V.I., Grigor'ev, A.M. J. Anal. Chem., 2004, Vol. 59, pp. 270-274. https://link.springer.com/article/10.1023/B:JAN C.0000018972.54587.ce.

3. Deineka V.I., Deineka L.A., Saenko I.I. $J$. Anal. Methods Chem., 2015, Vol. 2015, ID 732918. https://doi.org/10.1155/2015/732918.

4. Clifford M.N. J. Sci. Food Agric., 2000, Vol. 80, pp. 1033-1042. https://doi.org/10.1002/(SICI)10970010(20000 515)80:7<1033::AID-JSFA595>3.0.CO;2-T

5. IUPAC Commission on the Nomenclature of Organic Chemistry (CNOC) and IUPACIUB Commission on Biochemical Nomenclature $(\mathrm{CBN})$. Nomenclature of cyclitols. Recommendations, 1973, Biochem. J., 1976, Vol. 153, pp. 23-31. https://doi.org/10.1042/ bj1530023

6. Clifford M.N., Johnston K.L., Knight S. et al., J. Agric. Food Chem. 2003, Vol. 51, pp. 2900-2911. https://doi.org/10.1021/jf026187q.

7. de Paula Lima J., Farah A., King B. et al., J. Agric. Food Chem., 2016, Vol. 64, pp. 23612370. DOI: 10.1021 acs.jafc.6b00276

Дейнека Виктор Иванович - профессор кафедры общей химии, д.х.н., Белгородский государственный национальный исследовательский университет, Белгород

Олейниц Елена Юрьевна - аспирант Белгородского государственного национального исследовательского университета, Белгород

Дейнека Людмила Александровна - доцент кафедры общей химии, к.х.н., Белгородский государственный национальный исследовательский университет, Белгород
8. Schütz K., Kammerer D., Carle R., Schrieber A. J. Agric. Food Chem. 2004, Vol. 52, pp. 4090-4096. https://doi.org/10.1021/ jf049625x

9. Deineka V.I., Oleinits E.Yu., Blinova I.P. et al., J. Anal. Chem., 2019, Vol. 74, No 8, pp. 778-783. https://link.springer.com/article/ 10.1134/S1061934819080057

10. Schoenmakers P.J., Billiet H.A.H., Tijssen R. et al., J. Chromatogr. A, 1978, Vol. 149, pp. 519-537. https://doi.org/10.1016/S00219673(00)81008-0.

11. Murugesu K., Saghir S.A.M., Sadikun A. et al., Acta Chromatographica, 2021, Vol. 33, pp. 170-178. https://doi.org/10.1556/1326.2020. 00690 .

12. Bicchi C.P., Binello A.E., Pellegrho G.M., et al., J. Agric. Food Chem., 1995, Vol. 43, pp. 1549-1555. https://doi.org/10.1021/jf00054a 025 .

13 Ky C.-L., Noirot M., Hamon S. J. Agric. Food Chem., 1997, Vo. 45, pp. 786-790. https://doi.org/10.1021/jf9605254.

14. Xue M., Shi H., Zhang J., Liu Q.-Q. et al. Molecules, 2016, Vol. 21, 948. https://doi.org/ 10.3390/molecules 21070948

15. Stalmach A., Mullen W., Nagai C. et al. Braz. J. Plant Physiol., 2006, Vol. 18, pp. 253262. https://doi.org/10.1590/S1677-04202006 000100018 .

Deineka Viktor I. - Professor of the Department of General Chemistry Belgorod state national research University, Belgorod, e-mail deineka@,bsu.edu.ru

Oleinits Elena Yu. - student of Belgorod state national research University, Belgorod, e-mail oleinits_e@bsu.edu.ru

Deineka Lyudmila A. - Associate Professor of the Department of General Chemistry Belgorod state national research University, Belgorod, email deyneka@,bsu.edu.ru 